and investigate variations of lipid, glucose, and vascular endothelial growth factor (VEGF) levels myocardial perfusion images and cardiac function, as well as the possible mechanisms to improve myocardial perfusion.

Methods Total 102 cases were selected, 5 patients were lost. Finally 97 patients included, 77 males and 20 females, aged 56 \pm 13 . Of whom, 30 cases suffered from acute myocardial infarction (AMI), 48 from acute coronary syndromes (ACS), and 19 from chronic stable angina pectoris. They were randomised into three groups, $A(n=33)$, $B(n=31)$, and $C(n=33)$ without differences in the baseline level. Normal adults were control group $\mathrm{D}(\mathrm{n}=30)$. Groups $\mathrm{A}$ and $\mathrm{B}$ were given by Acarbose $50 \mathrm{mg}$ tid and $100 \mathrm{mg}$ tid, respectively, Groups C and $\mathrm{D}$ were given by placebo, the treatment course lasted three months, and CHD patients of each group underwent the same basic treatments after PCI. Myocardial perfusion imaging and variations of blood lipid, IGT, VEGF levels, myocardial perfusion images and cardiac function were observed.

Results 1 . The IGT had no statistically differences before treatments in A, B and C groups ( $p>0.05$ ). The changes of FPG and OGTT $2 \mathrm{~h}$ PG levels had statistically differences after treatments in $A$ and $B$ groups $(p<0.05)$, especially $B$ group $(p<0.01)$; The changes of HbA1c had no statistically significant differences after treatments in the four groups ( $p>0.05)$; The changes of lipid levels had no statistically differences $(p>0.05)$ before and after treatments in A, B, C and $\mathrm{D}$ groups, respectively. 2. A, B, C groups had the significantly higher plasma VEGF levels $(203 \pm 89 \mathrm{ng} / \mathrm{l}$ vs $77 \pm 52 \mathrm{ng} / \mathrm{l}, \mathrm{p}<0.01$ ) than $\mathrm{D}$ group before treatments. The changes of VEGF levels had statistically differences after treatments in $A$ and $B$ groups $(p<0.05)$, especially B group $(p<0.01)$. The changes of VEGF levels had no statistically differences before and after treatments in $C$ and $D$ groups $(p>0.05)$. 3. The myocardial perfusion images and cardiac function had no statistically differences before treatments in A, B and $C$ groups ( $p>0.05$ ), The changes of myocardial perfusion images had statistically differences after treatments in A and B groups $(p<0.05)$, especially B group $(p<0.01)$; The changes of cardiac function had statistically differences after treatments in $A$ and $B$ groups $(p<0.05)$, there is no statistically differences after treatments between $A$ and $B(p>0.05)$; The changes of myocardial perfusion images and cardiac function had no statistically differences in $\mathrm{C}$ group ( $p>0.05)$.

Conclusions Acarbose can regulates IGT, improve myocardial perfusion images and cardiac function. The mechanisms may include reducing VEGF levels, suppressing endothelial hyperplasia, and improving the microcirculation.

\section{e0343 TRIPLE VERSUS DUAL ANTIPLATELET THERAPY IN PATIENTS WITH ACUTE CORONARY SYNDROME UNDERGOING PERCUTANEOUS CORONARY INTERVENTION}

doi:10.1136/hrt.2010.208967.343

Bin Hu, Yujie Zhou, Yuyang Liu, Dongmei Shi, Yingxin Zhao, Dean Jia. Beijing Anzhen Hospital

Background Following percutaneous coronary intervention (PCI), clopidogrel in addition to aspirin therapy leads to greater protection from thrombotic complications than aspirin alone. Whether triple antiplatelet therapy is superior or similar to dual antiplatelet therapy in patients with acute coronary syndrome undergoing PCI in the era of drug-eluting stents remains unclear.

Objectives To evaluate the effect of triple antiplatelet vs dual antiplatelet therapy in patients with acute coronary syndrome after PCI.

Methods and Results We collected consecutive 1203 acute coronary syndrome patients undergoing drug-eluting stents implantation. They received either dual (aspirin plus clopidogrel; dual group; $\mathrm{n}=682$ ) or triple (aspirin plus clopidogrel plus cilostazol; triple group; $n=521$ ) antiplatelet therapy. The triple group received additional cilostazol at least for 1 month. Various major adverse cardiac events at 1 year were compared between these 2 groups. Compared with the dual group, the triple group had a similar incidence of major bleeding events but a significantly lower incidence of inhospital mortality. Clinical outcomes at 1 year showed that the triple group had significantly lower incidences of cardiac death and total major adverse cardiac events than the dual group.

Conclusions Triple antiplatelet therapy seems to be superior to dual antiplatelet therapy in patients ACS undergoing PCI with drugeluting stents.

\section{e0344 THE MECHANISM RESEARCH OF FRP INHIBITS ENDOTHELIAL CELL APOPTOSIS}

doi:10.1136/hrt.2010.208967.344

Shen Hua, Zhou Yujie, Liu Yuyang, Yang Shiwei, Gao Fei, Wang Zhijian, Shi Dongmei, Han Hongya, Ge Hailong, Liu Xiaoli. Department of Cardiology Beijing Anzhen Hospital Capital Medical University Beijing, China

Background Atherosclerosis is the most common cause of cardiovascular diseases in the world. Although the development of atherosclerosis appears to be the result of multiple maladaptive pathways, a particularly important factor in the pathogenesis of atherosclerosis is oxidised low density lipoprotein (ox-LDL), which contributes to endothelial damage. Data from our lab and others show that Follistatin related protein (FRP), which is expressed in the vasculature, has cardioprotective effects, suggesting that loss of FRP protection might play a role in the development of atherosclerosis. Objective In the present study, we determined whether FRP overexpression protects against endothelial cell (EC) damage, an intermediate endpoint for atherosclerosis.

Methods We bred ApoE knockout (ApoE (-/-)) mice that were FRP+ transgenic (they overexpressed FRP. We compared them to control mice (their littermates). Human umbilical vein endothelial cells (HUVECs) were isolated and treated with ox-LDL and recombinant FRP. FRP-induced signal transduction and $\mathrm{Bc} 2 \mathrm{mRNA}$ and protein stability were analysed.

Results After 16 weeks, ApoE (-/-) FRP (+) mice had significantly fewer apoptotic endothelial cells than controls. In vitro experiments showed that the effect of FRP on EC apoptosis was mediated by upregulation of expression of the antiapoptotic protein $\mathrm{Bcl} 2$.

Conclusion FRP overexpression maintains EC viability by preventing apoptosis via $\mathrm{Bcl} 2$ upregulation. FRP may be a novel therapeutic target for the prevention and treatment of vascular EC injury and of atherosclerosis.

\section{Q0345 SERUM LIPOPROTEIN (A) IS POSITIVELY CORRELATED WITH CORONARY ARTERY CALCIFICATION IN LOW RISK CHINESE PATIENTS}

doi:10.1136/hrt.2010.208967.345

Chengxing Shen, Jun Bao, Xiaoxia Chen, Genshan Ma. Zhongda Hospital southeast University

Background Prior studies indicated that lipoprotein (a) is an independent risk factor for coronary atherosclerosis, but the relationship of serum lipoprotein (a) and coronary artery calcification is still poorly understood in Chinese population.

Objective The present study is to investigate the human lipid profile of a single center (lipoprotein (a), other blood lipid levels) with the relationship of coronary artery calcification.

Method 388 patients suspected with coronary artery disease under coronary CT examinations from March 2007 to June 2009 in our 